\title{
EXTREME MAXIMAL IDEALS OF A PARTIALLY ORDERED VECTOR SPACE
}

\author{
F. F. BONSALL
}

1. Let $V$ be a partially ordered vector space with an order unit $e$. It is well known that the class $\mathfrak{M}$ of maximal ideals of $V$ is in one-one correspondence with the class $K$ of normalized positive linear functionals, in the sense that to each $M \in \mathfrak{M}$ corresponds a positive linear functional $\phi_{M}$ with $M$ as its null-space and with $\phi_{M}(e)=1$. A maximal ideal $M \in \mathfrak{M}$ is said to be extreme if $\phi_{M}$ is an extreme point of the convex set $K$; i.e., if the equation $\phi_{M}=\sigma \phi_{1}+(1-\sigma) \phi_{2}$ with $\phi_{1}, \phi_{2} \in K$ and $0<\sigma<1$ can hold only with $\phi_{1}=\phi_{2}=\phi_{M}$.

Kadison [2] has fully demonstrated the importance of the extreme maximal ideals in the theory of the representation of partially ordered vector spaces by function spaces. The main purpose of this article is to give a simple and direct characterization of the extreme maximal ideals in terms of the order structure in $V$. This characterization improves our understanding of the place of the extreme maximal ideals in the representation theory. For example, it becomes almost obvious that, if $V$ is lattice ordered, then the extreme maximal ideals coincide with the lattice maximal ideals.

We consider a class of ideals that we call "perfect" ideals. Besides providing the required characterization of the extreme maximal ideals as perfect maximal ideals, perfect ideals are of interest in their own right. The class of all perfect ideals has the most important properties of the class of all ideals. In particular, if $V$ has no perfect proper ideals other than (0), then $V$ is isomorphic to $R$.

The fundamental theorem on extreme points of convex sets of linear functionals is the Krein-Milman existence theorem [3]. This theorem is shown to be a simple consequence of the fact that each perfect proper ideal is contained in a perfect maximal ideal. In fact, the Krein-Milman theorem is related to this property of perfect ideals in the same way that the Hahn-Banach theorem is related to the corresponding property of ideals in general.

2. Definitions and notation. Let $V$ be a partially ordered vector space, namely a vector space over $R$ in which a nonempty positive cone $\mathrm{V}^{+}$is specified having the following properties:

Presented to the Society, October 22, 1955; received by the editors August 1, 1955 and, in revised form, October 7, 1955. 
(i) if $x, y \in V^{+}$and $\alpha \geqq 0$, then $x+y, \alpha x \in V^{+}$;

(ii) if $x,-x \in V^{+}$, then $x=0$.

We write $x \geqq y$ (or $y \leqq x$ ) to denote that $x-y \in V^{+}$.

Let $V$ have an order unit e, namely an element of $V^{+}$such that, for each $x \in V$, there exists $\xi \in R$ with

$$
x \leqq \xi e .
$$

An ideal of $V$ is a subspace $J$ such that $J$ contains all elements $x$ that satisfy the inequalities $-j \leqq x \leqq j$ for some $j \in J$. An ideal $J$ is called proper if $J \neq V$. It is well known $[1 ; 2]$ that each proper ideal of $V$ is contained in a maximal ideal (i.e. a maximal proper ideal).

A positive linear functional is a nonzero linear functional $\phi$ on $V$ such that $\phi(x) \geqq 0$ whenever $x \geqq 0$. A normalized positive linear functional (n.p.l.f.) is a positive linear functional $\phi$ with $\phi(e)=1$. The class of all n.p.l.f.s is denoted by $K$. To each maximal ideal $M$ corresponds a unique n.p.l.f. $\phi_{M} \in K$ such that $M$ is the null-space of $\phi_{M}$. Conversely, the null-space of each positive linear functional is a maximal ideal. Extreme maximal ideals have been defined in $\$ 1$ above.

Given a subspace $V_{0}$ of $V$, let $H\left(V_{0}\right)$ denote the set of all elements $x \in V$ such that, for every positive real number $\epsilon$, there exists an element $w_{\epsilon} \in V_{0}$ satisfying the inequalities

$$
-\left(\epsilon e+w_{\epsilon}\right) \leqq x \leqq \epsilon e+w_{\epsilon} .
$$

An ideal $J$ of $V$ is said to be perfect if

$$
J \subset H(J) \text {. }
$$

It should be noted that an ideal $J$ of $V$ is perfect if and only if, for each $x$ in $J$ and $\epsilon>0$, there exists an element $w_{\epsilon}$ in $J$ such that $0 \leqq \epsilon e+w_{\epsilon}$ and $x \leqq \epsilon e+w_{\epsilon}$. In fact, if $J$ has this property, $x \in J$, and $\epsilon>0$, then there exist $u, v \in J$ with $\left.{ }_{0}^{x}\right\} \leqq(\epsilon e / 2)+u$ and $\left.{ }_{0}^{-x}\right\} \leqq(\epsilon e / 2)+v$; and therefore $\pm x \leqq \epsilon e+(u+v)$. This shows that the condition is sufficient, and its necessity is obvious.

It should also be remarked that the class of perfect ideals is independent of the choice of order unit $e$ used in their definition. In fact, if $f$ is another order unit of $V$, then there exist positive constants $\xi$ and $\eta$ such that $e \leqq \xi f$ and $f \leqq \eta e$.

\section{Characterization of extreme maximal ideals.}

Theorem 1. A maximal ideal of $V$ is extreme if and only if it is perfect.

Proof. (A). Let $M$ be a perfect maximal ideal, let $\phi_{M}$ be the cor- 
responding n.p.l.f., and let $\phi_{M}=\sigma \phi_{1}+(1-\sigma) \phi_{2}$ with $\phi_{1}, \phi_{2} \in K$ and $0<\sigma<1$. If $x \in M$, then $x \in H(M)$, and therefore, for each $\epsilon>0$, there exists an element $m_{\epsilon} \in M$ such that

$$
-\left(\epsilon e+m_{\epsilon}\right) \leqq x \leqq \epsilon e+m_{\epsilon} .
$$

Since $\epsilon e+m_{\epsilon} \geqq 0$, we have $\phi_{i}\left(\epsilon e+m_{\epsilon}\right) \geqq 0(i=1,2)$. Also, since $m_{\epsilon} \in M$,

$$
\epsilon=\phi_{M}\left(\epsilon e+m_{\epsilon}\right)=\sigma \phi_{1}\left(\epsilon e+m_{\epsilon}\right)+(1-\sigma) \phi_{2}\left(\epsilon e+m_{\epsilon}\right) \text {. }
$$

It follows that

$$
0 \leqq \phi_{1}\left(\epsilon e+m_{\epsilon}\right) \leqq \epsilon / \sigma
$$

and therefore

$$
\pm \phi_{1}(x) \leqq \epsilon / \sigma .
$$

Since $\epsilon$ is an arbitrary positive number, we have $\phi_{1}(x)=0$, and therefore the null-space of $\phi_{1}$ coincides with that of $\phi_{M}$. This proves that $\phi_{1}=\phi_{M} ; \phi_{M}$ is an extreme point of $K$.

(B). Let $M$ be an extreme maximal ideal, and let $\phi_{M}$ be the corresponding n.p.l.f. We consider the functional $q$ defined on $V$ by

$$
q(x)=\inf \left[\phi_{M}(a): a \in V^{+}, a \geqq x\right] .
$$

It is easily verified that $q$ has the following properties:

[3.1] $q$ is sublinear (i.e. subadditive and positive-homogeneous);

[3.2] if $x \leqq 0$, then $q(x)=0 ; q(y) \geqq 0$ for all $y$ in $V$;

[3.3] if $x \geqq 0$, then $q(x)=\phi_{M}(x)$.

We prove:

[3.4] if $x \in M$, then $q(x)=0$.

By [3.1], given $m \in M$, there exists a linear functional $\psi$ with $\psi(m)=q(m)$ and $\psi(x) \leqq q(x)(x \in V)$. By [3.2], $\psi$ is a non-negative ${ }^{1}$ linear functional. By $[3.3], \psi(x) \leqq \phi_{M}(x)$ whenever $x \geqq 0$. It follows that $\phi_{M}-\psi$ is a non-negative linear functional. Let $\alpha=\phi_{M}(e)-\psi(e)$. If $\alpha=0$, then $\phi_{M}-\psi=0, q(m)=\psi(m)=\phi_{M}(m)=0$. If $\alpha=1$, then $\psi(e)=0, \psi=0, q(m)=\psi(m)=0$. Finally, if $0<\alpha<1$, then the equation

$$
\phi_{M}=\alpha \psi_{1}+(1-\alpha) \psi_{2}
$$

where $\psi_{1}=\left(\phi_{M}-\psi\right) / \alpha$ and $\psi_{2}=\psi /(1-\alpha)$, expresses $\phi_{M}$ as a convex combination of n.p.l.f.s. Since $\phi_{M}$ is extreme, we conclude that $\phi_{M}$ $=\psi_{2}$, and therefore

$$
q(m)=\psi(m)=(1-\alpha) \phi_{M}(m)=0 .
$$

This completes the proof of [3.4].

1 Non-negative on $\mathrm{V}^{+}$. 
We now complete the proof that $M$ is perfect. Let $m \in M$ and $\epsilon>0$. By [3.4], there exists an element $a \in V^{+}$such that $m \leqq a$ and $\eta=\phi_{M}(a)$ $<\epsilon$. Let $b_{\mathrm{e}}=a-\eta e$. Then

$$
\phi_{M}\left(b_{\varepsilon}\right)=\phi_{M}(a)-\eta=0,
$$

and therefore $b_{\epsilon} \in M$. Also

$$
b_{\epsilon}+\epsilon e \geqq b_{\epsilon}+\eta e=a \geqq\left\{\begin{array}{l}
m, \\
0 .
\end{array}\right.
$$

Thus $M$ is perfect, and the proof is complete.

CoRollary. If $V$ is lattice ordered, then a maximal ideal of $V$ is extreme if and only if it is a lattice ideal.

Note. $V$ is said to be lattice ordered if, given $x, y \in V$, there exists an element $z=\max (x, y)$ such that $z \geqq\left\{\begin{array}{l}x \\ y\end{array}\right.$, and if $z^{\prime} \geqq\left\{\begin{array}{l}x \\ y\end{array}\right.$, then $z^{\prime} \geqq z$. The element $\max (x,-x)$ is denoted by $|x|$. An ideal $J$ of $V$ is called a lattice ideal if $|x| \in J$ whenever $x \in J$.

ProOF OF THE COROLLARY. (A). Let $M$ be a lattice maximal ideal. If $m \in M$, then $|m| \in M$ and

$$
-|m| \leqq m \leqq|m| .
$$

This shows that $m \in H(M)$, and therefore $M$ is perfect. By Theorem 1, $M$ is extreme.

(B). Let $M$ be an extreme maximal ideal, and let $\phi_{M}$ be the corresponding n.p.l.f. By Theorem 1, $M$ is perfect. Given $m \in M$ and $\epsilon>0$, there exists an element $m_{\epsilon} \in M$ such that $\pm m \leqq m_{\epsilon}+\epsilon e$. Since $|m|=\max (m,-m)$, it follows that

$$
0 \leqq|m| \leqq m_{\epsilon}+\epsilon e .
$$

Since $\phi_{M}\left(m_{\epsilon}\right)=0$, this implies that

$$
0 \leqq \phi_{M}(|m|) \leqq \phi_{M}\left(m_{\epsilon}+\epsilon e\right)=\epsilon .
$$

Since $\epsilon$ is an arbitrary positive number, this shows that $\phi_{M}(|m|)=0$, $|m| \in M$. Thus $M$ is a lattice ideal.

4. Properties of perfect ideals. The following propositions are almost obvious.

[4.1] If $Y$ is a subspace of $V$, then $H(Y)$ is an ideal.

[4.2] If $Y$ is a subspace and $Y \subset H(Y)$, then $H(Y)$ is a perfect ideal.

[4.3] If $M$ is a maximal ideal containing the subspace $Y$, then $H(Y) \subset M$. (Consider a positive linear functional with $M$ as its nullspace.) 
TheOREM 2. If (0) is the only proper perfect ideal of $V$, then $V=(e)$, the one-dimensional space spanned by $e$.

Proof. Let $p$ be the functional defined on $V$ by

$$
p(x)=\inf [\xi: \xi e \geqq x] .
$$

The following propositions are easily verified:

[4.5] $p$ is sublinear on $V$;

[4.6] $p(e)=-p(-e)=1$;

[4.7] $p(x) \leqq 0$ whenever $x \leqq 0$.

Given $u \in V$, let $y=p(u) e-u$. By [4.5], there exists a linear functional $\phi$ defined on $V$ with $\phi(u)=p(u)$ and $\phi(x) \leqq p(x)(x \in V)$. By [4.6] and [4.7], $\phi$ is an n.p.l.f. Also

$$
\phi(y)=p(u)-\phi(u)=0 .
$$

By definition of $p$, for each $\epsilon>0$, we have $(\epsilon e / 2)+y \geqq 0$. Thus

$$
-(\epsilon e+y) \leqq y \leqq \epsilon e+y \text {. }
$$

Let $Y=(y)$, the one-dimensional space spanned by $y$. Then (4.9) shows that $y \in H(Y)$, and therefore

$$
Y \subset H(Y) \text {. }
$$

If $y$ is nonzero, then, by (4.10) and [4.2], $H(Y)$ is a nonzero perfect ideal of $V$. By (4.8), $Y$ is contained in the null-space of $\phi$, and therefore, by [4.3], $H(Y)$ is contained in the null-space of $\phi ; H(Y)$ is proper.

We have now proved that, if $y \neq 0$, then $H(Y)$ is a nonzero proper perfect ideal. Hence $y=0, u=p(u) e, V=(e)$.

TheOREM 3. If $J$ is a perfect proper ideal of $V$, then $J$ is contained in a perfect maximal ideal of $V$.

COROLlaRY. $J$ is contained in the null-space of an extreme point of $K$.

Proof. Let $J$ be a proper perfect ideal of $V$, and consider the class of all proper perfect ideals $F$ of $V$ that contain $J$. By Zorn's lemma, there exists a maximal subclass $\&$ that is simply ordered by the relation of inclusion. Let

$$
M=\bigcup_{F \in \mathbb{E}} F
$$

It is easily verified that $M$ is a proper perfect ideal of $V$, and obviously $M$ contains $J$. 
We prove that the only proper perfect ideal of the partially ordered vector space $^{2} V / M$ is (0). In fact, let $G$ be a proper perfect ideal of $V / M$ and let

$$
F=[x: x \in V,[x \in G],
$$

where $[x]$ denotes the coset of $x$ in $V / M$. We show that $F$ is a perfect ideal of $V$.

Let $x \in F$. Then $[x] \in G \subset H(G)$, and therefore, for every $\epsilon>0$, there exists an element $\left[y_{\mathrm{e}}\right] \in G$ with

$$
-\left(\left[y_{\epsilon}\right]+\epsilon[e]\right) \leqq[x] \leqq\left[y_{\epsilon}\right]+\epsilon[e] .
$$

By definition of the order relation in $V / M$, there exist elements $f_{\epsilon}, g_{e}$ in $M$ such that

$$
\begin{array}{r}
x \leqq y_{\epsilon}+f_{\epsilon}+\epsilon e, \\
-x \leqq y_{\epsilon}+g_{\epsilon}+\epsilon e .
\end{array}
$$

Since $M$ is a perfect ideal, there exist elements $h_{\epsilon}, k_{\epsilon}$ in $M$ such that

$$
\begin{aligned}
& -\left(h_{\epsilon}+\epsilon e\right) \leqq f_{\epsilon} \leqq h_{\epsilon}+\epsilon e, \\
& -\left(k_{\epsilon}+\epsilon e\right) \leqq g_{\epsilon} \leqq k_{\epsilon}+\epsilon e .
\end{aligned}
$$

Since $h_{\epsilon}+\epsilon e \geqq 0$ and $k_{\epsilon}+\epsilon e \geqq 0$, we have

$$
\left.\begin{array}{l}
f_{\epsilon} \\
g_{\epsilon}
\end{array}\right\} \leqq h_{\epsilon}+k_{\epsilon}+2 \epsilon e,
$$

and therefore

$$
\pm x \leqq y_{\mathrm{e}}+h_{\mathrm{e}}+k_{\mathrm{e}}+3 \epsilon e .
$$

Since $\epsilon$ is an arbitrary positive number and $y_{\epsilon}+h_{\epsilon}+k_{\epsilon} \in F$, these last inequalities show that $x \in H(F)$.

We have now proved that $F$ is a proper perfect ideal of $V$, and, since $F \supset M$, the maximal property of $\&$ implies that $F=M$. This proves that $V / M$ has no proper perfect ideals other than $(0)$, and therefore, by Theorem $2, V / M=([e])$, the one-dimensional space spanned by $[e]$. Consequently, $M$ is a maximal ideal of $V$.

THEOREM 4. (Krein-Milman). Let $p$ be a sublinear functional on a vector space $W$ over $\boldsymbol{R}$, and let $H$ denote the convex set of all linear functionals $\phi$ defined on $W$ such that $\phi(x) \leqq p(x)(x \in W)$. If $x_{0} \in W$, then there exists an extreme point $\phi_{0}$ of $H$ such that $\phi_{0}\left(x_{0}\right)=p\left(x_{0}\right)$.

2 If $E$ is an ideal of $V$, then the quotient space $V / E$ becomes a partially ordered vector space if $(V / E)^{+}$is taken to be the set of cosets $[x]$ that contain elements of $V^{+}$. $V / E$ has an order unit $[e]$. 
Note. This slightly generalized form of the Krein-Milman theorem is proved in [1], where its relation to the original form [3] is stated. The proof given below follows the same general lines as the proof of the Hahn-Banach theorem given in [1].

Proof. Let $V$ denote the vector space $W+R$ of all ordered pairs $(x, \xi)$ with $x \in W$ and $\xi \in R$, with the natural definitions of the vector space operations. Let $e=(0,1)$, and denote $(x, \xi)$ by $x+\xi e . V$ becomes a partially ordered vector space, with order unit $e$, if we take $V^{+}$to consist of $0=(0,0)$ together with all $x+\xi e$ such that $\xi>p(-x)$. We extend $p$ to the whole of $V$ by the definition

$$
p(x+\xi e)=p(x)+\xi .
$$

It is easily verified that $p$ satisfies (4.4) in $V$.

Given $x_{0} \in W$, let $u=x_{0}-p\left(x_{0}\right) e$ and $y=p(u) e-u$. Clearly, $y=p\left(x_{0}\right) e-x_{0}$. We have seen, in the proof of Theorem 2, that, if $Y=(y)$, then $H(Y)$ is a proper perfect ideal of $V$, and $Y \subset H(Y)$. By the corollary to Theorem 3, there exists an extreme n.p.l.f. $\phi$ with null-space $M$ containing $H(Y)$.

Let $\phi_{0}$ be the functional defined on $W$ by $\phi_{0}(x)=\phi\{(x, 0)\}(x \in W)$. It is easily verified that $\phi_{0} \in H$, and, since $p\left(x_{0}\right) e-x_{0}=y \in M$, that $\phi_{0}\left(x_{0}\right)=p\left(x_{0}\right)$. Finally, $\phi_{0}$ is an extreme point of $H$. For, if $\psi_{0} \in H$ and $\psi$ is the functional defined on $V$ by $\psi(x+\xi e)=\psi_{0}(x)+\xi$, then $\psi$ is an n.p.l.f.

I am indebted to the referee for correcting an error in the proof of Theorem 1.

\section{REFERENCES}

1. F. F. Bonsall, Sublinear functionals and ideals in partially ordered vector spaces' Proc. London Math. Soc. vol. 4 (1954) pp. 402-418.

2. R. V. Kadison, A representation theory for commutative topological algebra, Memoirs of the American Mathematical Society vol. 7 (1951).

3. M. Krein and D. Milman, On extreme points of regular convex sets, Studia Mathematica vol. 9 (1940) pp. 133-138.

Durham University, NewCastle-on-Tyne 